\title{
Sterben in Würde
}

Zur Sterbehilfedebatte aus katholischer Perspektive

\author{
GERHARD MARSCHÜTZ*
}

To die in dignity is often understood as a personal right to independently determine the time and the way of one's own death, assuming dignity as a quality of life that wouldn't exist in serious illness or in the final stage of life. On the contrary and from a traditional point of view dignity belongs to a person's being that can never get lost and hence has to be respected also at the end of life. For that reason, from a catholic point of view a voluntary euthanasia as a deliberate killing of a person is definitely rejected. However, a so-called 'aggressive medical treatment' must be avoided because it doesn't accept the actual situation of death and only causes a pointless extension of life. Nevertheless, the palliative care is essential to provide people even in their final lifetime with quality of life and to help them with the personal acceptance of death.

Keywords: To die in dignity, dignity, euthanasia, Catholic Church, evangelium vitae, palliative care, theological ethics

Zum Leben gehört auch das Sterben. Somit umfasst die Würde des Menschen auch sein Sterben als letzte Phase des Lebens. Doch was besagt ein Sterben in Würde? Impliziert es auch die Tötung auf Verlangen oder steht ein solches Handeln der Menschenwürde entgegen? Das sind schwierige Fragen, die strittig diskutiert werden und auf die es offenbar keine einfachen Antworten gibt. Zwar mangelt es nicht an einfachen Antworten, die aber zumeist nur einen wenig hilfreichen Entweder-Oder-Diskurs bewirken. „Unmenschlich am Lebensende“ verhalte sich dann in einem solchen Diskurs selbst die katholische Kirche, da sie „das Selbstbestimmungsrecht mit Verweis auf die Gottgegebenheit allen menschlichen Lebens [relativiert] “" , meint etwa der Berliner Notfall- und Intensivmediziner Michael de Ridder. Umgekehrt erweisen sich für Papst Johannes Paul II. die modernen Bemühungen um die Legalisierung der freiwillige Euthanasie, „wenn man es zutiefst betrachtet, als absurd und unmenschlich" (EV 64). Was ist, wenn man es zutiefst betrachtet, menschlich oder unmenschlich am Le-

\footnotetext{
Gerhard Marschütz, Ao. Univ.-Prof. Dr. an der Katholisch-Theologischen Fakultät der Universität Wien, Institut für Systematische Theologie, Fachbereich Theologische Ethik. Adresse: A-1010 Wien, Schenkenstraße 8-10; e-mail: gerhard.marschuetz@univie.ac.at.

1 Michael de Ridder, Wie wollen wir sterben? Ein ärztliches Plädoyer für eine neue Sterbekultur in Zeiten der Hochleistungsmedizin, München 52011, S. 200.
}

RES 6 (2/2014), p. 174-196

DOI: $10.2478 /$ ress-2014-0115 
bensende? Worin besteht ein menschenwürdiges, worin ein menschenunwürdiges Sterben? ${ }^{2}$

\section{Selbstbestimmtes oder verfügtes Sterben?}

„Gehört es auch zum menschenwürdigen Sterben, dass der Mensch selber [...] über Zeitpunkt und Art und Weise des Sterbens befinden kann?"“3 Auf diese Frage spitzt sich für den katholischen Theologen Hans Küng die gegenwärtige Sterbehilfedebatte zu, wenn es um die Menschenwürde am Lebensende und somit um das Selbstbestimmungsrecht alter, kranker, pflegebedürftiger und sterbebereiter Menschen geht. In seinem gemeinsam mit dem Literaturwissenschaftler Walter Jens herausgegebenen Buch Menschenwürdig sterben. Ein Plädoyer für Selbstverantwortung bejaht er diese Frage. Wenn ein Mensch kontinuierlich keinerlei Hoffnung auf ein humanes Weiterleben mehr sieht, dann soll aktive Sterbehilfe auf dessen Wunsch hin als ultimative Lebenshilfe nicht verweigert werden.

Dieses Buch hat Mitte der 1990er Jahre lebhafte Diskussionen ausgelöst, insbesondere im katholischen Bereich, da ein Plädoyer für selbstbestimmtes Sterben bis dahin nur von säkularen Autoren vorgetragen wurde. Küng und Jens wollten mit ihrer Veröffentlichung das damals in Deutschland weitgehend tabuisierte und in der medizinischen Praxis in einer Grauzone belassene Thema der freiwilligen aktiven Sterbehilfe „auf ein anderes ethisches Niveau heben“, um „nüchtern, würdig und moralisch-ernsthaft“ die Frage nach der "Selbstverantwortung des Menschen für sein Sterben“ neu thematisieren zu können. ${ }^{4}$ Neu stellt sich diese Frage nicht nur angesichts der modernen Möglichkeiten einer Hochleistungsmedizin, welche das menschliche Sterben oft sinnlos verlängern und somit das Lebensrecht zum Lebenszwang werden lassen. Auch aus theologischer Sicht ist es für Küng im Verständnis einer theonom begründeten Autonomie angezeigt, dass „dem Menschen ein Selbstbestimmungsrecht auch im Sterben zukommt" ${ }^{\text {"s }}$. Er sucht daher

„für einen theologisch und christlich verantworteten Weg der Mitte einzutreten: zwischen einem antireligiösen Libertinismus ohne Verant-

2 Vgl. hierzu: Matthias Mettner (Hg.), Wie menschenwürdig sterben? Zur Debatte um die Sterbehilfe und zur Praxis der Sterbebegleitung, Zürich ${ }^{3} 2003$; Stephan E. Müller, Rainer Beckmann (Hg.), Menschenwürdig sterben - aber wie? Medizinische, juristische und ethische Aspekte, Glaube und Ethos Bd. 9, Berlin, 2010.

3 Walter Jens, Hans Küng (Hg.), Menschenwürdigsterben. Ein Plädoyerfür Selbstverantwortung, München 1995, S. 49.

4 Ibidem, S. 11.

5 Ibidem, S. 66. 
wortung (,unbeschränktes Recht auf den Freitod') und einem reaktionären Rigorismus ohne Mitleid (,auch Unerträgliches ist als gottgegeben gottergeben zu ertragen'). Und ich tue dies, weil ich als Christ und Theologe der Meinung bin: Der allbarmherzige Gott, der den Menschen Freiheit geschenkt hat und Verantwortung für sein Leben zugemutet hat, hat gerade auch dem sterbenden Menschen die Verantwortung und Gewissensentscheidung für Art und Zeitpunkt seines Todes überlassen." 6

Diesen für sterbenskranke, aber auch für sterbenswillige Menschen zu öffnenden Weg der Mitte will Küng in Anlehnung an die niederländische Praxis durch eine neue gesetzliche Regelung der Sterbehilfe realisiert sehen, welche Ärzten unter bestimmten Bedingungen eine Tötung auf Verlangen ermöglicht.

Jens bekräftigt das Anliegen eines selbstbestimmten Sterbens anhand einer Vielfalt literarischer Texte, auf die hier nicht einzugehen ist. Erwähnenswert ist aber, dass Jens acht Jahre nach dem Erscheinen des Buches an vaskulärer Demenz erkrankt (2003) und ein Jahrzehnt danach 90-jährig am 9. Juni 2013 in Tübingen stirbt. Erwähnenswert deshalb, weil beide Autoren einander versprachen, dem anderen im Fall einer solchen Krankheit behilflich zu sein, den Wunsch des selbstbestimmten Sterbens einlösen zu können. Auch Jens Ehefrau akzeptierte dieses Versprechen. Dennoch vermochte sie es nicht einzulösen, da sich ihr Mann vor allem in früheren Phasen des Verlaufs seiner Krankheit immer wieder widersprüchlich äußerste. In einem Interview sagte Inge Jens:

„In lichten Momenten sagte mein Mann: ,Nicht totmachen, nicht totmachen', aber auch: ,ich will nicht mehr. Ich will sterben.' Als Gesunder hat er für die Sterbehilfe plädiert, und als Kranker hat er leben wollen. Mit dieser Erkenntnis bin ich noch lange nicht fertig. Doch wer hätte das Recht gehabt, ihn umzubringen? Ob ich richtig oder falsch entschieden habe, werde ich nie erfahren, damit muss ich leben. Ich weiß nicht, ob ich, wenn ich in dem Zustand sein werde, dann nicht auch leben will. Ich bin nicht besonders fromm, aber das muss ich einer mich übersteigenden Kraft anheimstellen. ${ }^{\text {"7 }}$

Im Kontext der Pflegeerfahrungen mit ihrem Mann war Inge Jens also herausgefordert, ihre ursprünglichen Vorstellungen einer Hilfe zum Sterben

6 Ibidem, S. $71 \mathrm{f}$.

7 http://www.zeit.de/2014/07/rettung-inge-jens/seite-2, abgerufen am 5. Juni 2014. 
zu revidieren und ihre Sicht von menschenwürdigen Leben und Sterben neu zu überdenken. Auch Hans Küng sah sich außer Stande sein Versprechen einzulösen - nicht nur wegen der fehlenden legalen Möglichkeit in Deutschland, sondern vor allem, weil ihm während der Krankheit seines Freundes Walter Jens immer deutlicher bewusst wurde,

„dass man angesichts eines Menschen, der keine körperlichen, sondern nur seelische Schmerzen erfährt, sich nicht befugt sehen kann einzugreifen, ja, dass unter Umständen nichts anderes übrigbleibt, als den Lauf des Geschehens einer anderen, höheren Instanz anheimzustellen“8.

Hätte Küng im Fall von körperlichen Schmerzen eine alternative Möglichkeit, etwa im benachbarten Ausland, in die Wege geleitet? Vermutlich hätte er diese Frage ebenso nicht eindeutig beantworten können, da hierfür die Art der Schmerzen und die Aussicht ihrer Linderung bekannt sein müssten, was selbst in der konkreten Situation oft nur bedingt fassbar wird.

Das Schicksal Jens' auf dem Hintergrund seines im Buch geäußerten Anliegens zeigt freilich, dass er auf eine Weise seinem Sterben entgegengegangen ist, wie er es aus der Sicht gesunder Tage nicht wollte. Es zeigt auch, dass er in der Krankheit zerrissen war zwischen Lebensbejahung und Sterbewunsch und der Lebenswille offensichtlich stärker war als der Todeswille. Damit zeigt sich ebenso, dass Verfügungen über das eigene Sterben im Vollbesitz der geistigen Kräfte nur relativ für die Krankheitssituation sind, da im Voraus niemals wissbar ist, mit welchen Beeinträchtigungen jemand auf keinen Fall leben kann. Das vorausverfügte selbstbestimmte Sterben unterstellt daher eine Sicherheit, die nicht typisch ist für die Situation, in der es eingelöst werden soll.

Wäre demnach der verfügte Tod „als gottgegeben gottergeben zu ertragen“ (Hans Küng)? Sind „zutiefst betrachtet“ (Johannes Paul II.) das eigene Sterben und der eigene Tod einer den Menschen „übersteigenden Kraft“ (Inge Jens) beziehungsweise einer „höheren Instanz anheimzustellen“ (Hans Küng)?

Aus katholischer Sicht stellt jedenfalls die in einigen Ländern bereits realisierte und in anderen vermehrt angestrebte Legalisierung eines selbstbestimmtes Sterbens, wie Papst Benedikt XVI. es prägnant formulierte, „eine menschenunwürdige Lösung" dar, da die wahre Antwort auf das Sterben von Menschen nur darin bestehen könne, „Zeugnis abzulegen für die Liebe, die hilft, dem Schmerz und dem Todeskampf auf menschliche Weise zu

http://www.faz.net/aktuell/feuilleton/der-fall-walter-jens-mich-erschuettert-diesermann-1771099.html, abgerufen am 5. Juni 2014. 
begegnen“". Auch Papst Johannes Paul II. sieht das „Drama der Euthanasie“ motiviert „von Gefühlen eines falsch verstandenen Mitleids oder einer missverstandenen zu bewahrenden Würde" ${ }^{10}$. Diese konträre Position der katholischen Kirche gründet in einem Denkrahmen, der nachfolgend kurz zu skizzieren ist.

Zunächst wird klar gesehen, dass angesichts des Todes „das Rätsel des menschlichen Daseins am größten "11 wird, insofern hier der Mensch

„nicht nur den Schmerz und den fortschreitenden Abbau des Leibes [erfährt], sondern auch, ja noch mehr die Furcht vor immerwährendem Verlöschen. Er urteilt daher im Instinkt seines Herzens richtig, wenn er die völlige Zerstörung und den endgültigen Untergang seiner Person mit Entsetzen ablehnt. Der Keim der Ewigkeit im Menschen lässt sich nicht auf die bloße Materie zurückführen und wehrt sich gegen den Tod. “12

Dieser Keim der Ewigkeit gründet in der Gottebenbildlichkeit des Menschen, darin, dass jeder Mensch von Gott her und auf Gott hin geschaffen ist. Hieraus ergibt sich ein Verständnis „des menschlichen Lebens als Leben der Beziehung, als Gottesgeschenk, als Frucht und Zeichen seiner Liebe"13.

Aus dieser relationalen Struktur der unbedingten Zuwendung Gottes zum Menschen folgt in kirchlichen Dokumenten als zentrale Prämisse bioethischen Nachdenkens: „das menschliche Leben, ein wertvolles Geschenk Gottes, ist heilig und unantastbar" ${ }^{\text {"14. }}$. Ethisch wird die Vorstellung von der „Heiligkeit des Lebens" durch das gleichermaßen religiös wie auch säkular begründbare Prinzip der Menschenwürde verdeutlicht. Diese kommt Würde in katholischer Sicht ,jedem Menschen von der Empfängnis bis zum natür-

9 Benedikt XVI., Angelus am 1.2.2009, http://www.vatican.va/holy_father/benedict_xvi/ angelus/2009/documents/hf_ben-Xvi_ang_20090201_ge.html, abgerufen am 5. Juni 2014 .

10 Ansprache von Johannes Paul II. an die Teilnehmer der 19. internationalen Konferenz des Päpstlichen Rats für die Krankenpastoral (12.11.2004), Nr. 3, http://www.vatican.va/ holy father/john_paul_ii/speeches/2004/november/documents/hf_jp-ii_spe_20041112 pc-hilthwork_ge.html, abgerufen am 5. Juni 2014.

11 Konzilskonstitution Gaudium et spes, Nr. 18, http://www.vatican.va/archive/hist councils/ii_vatican_council/documents/vat-ii_const_19651207_gaudium-et-spes_ ge.html, abgerufen am 5. Juni 2014.

12 Ibidem.

13 Johannes Paul II., Enzyklika Evangelium vitae (25.3.1995), Nr. 80, http://www. vatican.va/holy_father/john_paul_ii/encyclicals/documents/hf_jp-ii_enc_25031995 evangelium-vitae_ge.html, abgerufen am 5. Juni 2014.

14 Ibidem, Nr. 81. 
lichen Tod"15 zu. In sozialer Perspektive muss darum auch „die ganze Gesellschaft die Würde jeder menschlichen Person in jedem Augenblick und in jeder Lage ihres Lebens achten, verteidigen und fördern" ${ }^{16}$. Und weil auch das Sterben ein Teil des Lebens ist, wird ausdrücklich betont:

„Das Leiden, das Altern, der Zustand der Bewusstlosigkeit, der bevorstehende Tod verringern nicht die innewohnende Würde der Person, die als Abbild Gottes geschaffen ist." 17

Dem Geschenkcharakter menschlichen Daseins entspricht ferner, dass der Mensch ebenso wenig Herr über das Leben ist wie über den Tod. ${ }^{18}$ Deshalb gilt es den Tod - der für Christen nicht einfach nur das Ende, sondern zugleich Übergang zum ewigen Leben ist - „mit aller Würde“ anzunehmen, „ohne die Todesstunde in irgendeiner Weise zu beschleunigen “19. Folglich soll der Mensch in Würde sterben können, wann Gott es verfügt. Nicht der selbstbestimmte, sondern der natürlich bestimmte und in diesem Sinne verfügte Tod wahrt gemäß katholischer Auffassung ein Sterben in Würde.

Nur im Horizont dieser theologisch-ethischen Grundlagen des Nachdenkens über biomedizinische Herausforderungen wird die katholische Position zur Euthanasie verständlich.

15 Kongregation für die Glaubenslehre, Instruktion Diginatas personae (8.9.2008), Nr. 1. Es heißt hier: „Dieses Grundprinzip, das ein großes, Ja’ zum menschlichen Leben ausdrückt, muss im Mittelpunkt des ethischen Nachdenkens über die biomedizinische Forschung stehen.", http://www.vatican.va/roman_curia/congregations/cfaith/documents/rc_con_cfaith_ doc_20081208_dignitas-personae_ge.html, abgerufen am 5. Juni 2014.

16 Johannes Paul II., Enzyklika Evangelium vitae, Nr. 81.

17 Ansprache von Johannes Paul II., Nr. 3.

18 Vgl. Johannes Paul II., Enzyklika Evangelium vitae, Nr. 39: „Das Leben des Menschen kommt aus Gott, es ist sein Geschenk, sein Abbild und Ebenbild, Teilhabe an seinem Lebensatem. Daher ist Gott der einzige Herr über dieses Leben: der Mensch kann nicht darüber verfügen. [...] Leben und Tod des Menschen liegen also in den Händen Gottes, in seiner Macht: "In seiner Hand ruht die Seele allen Lebens und jeden Menschenleibes Geist», ruft Ijob aus (12,10). «Der Herr macht tot und lebendig, er führt zum Totenreich hinab und führt auch herauf» (1 Sam 2,6). Er allein kann sagen: "Ich bin es, der tötet und der lebendig macht» (Dtn 32,39). Aber diese Macht übt Gott nicht als bedrohliche Willkür aus, sondern als liebevolle Umsicht und Sorge gegenüber seinen Geschöpfen. Wenn es wahr ist, dass das Leben des Menschen in Gottes Händen ruht, so ist es ebenso wahr, dass es liebevolle Hände sind wie die einer Mutter, die ihr Kind annimmt, nährt und sich um es sorgt."

19 Kongregation für die Glaubenslehre, Erklärung zur Euthanasie (5.5.1980), Schluss, http://www.vatican.va/roman_curia/congregations/cfaith/documents/rc_con_cfaith_ doc_19800505_euthanasia_ge.httml, abgerufen am 5. Juni 2014. 


\section{Begriffliche Unterscheidungen des katholischen Lehramtes}

Das katholische Lehramt verwendet in ihren Dokumenten bewusst den - auch im internationalen Diskurs gebräuchlichen - Begriff Euthanasie, da der Begriff Sterbehilfe aufgrund seiner vieldeutigen Interpretationsmöglichkeit als problematisch angesehen wird. Dagegen wird im deutschsprachigen Raum wegen der aus der nationalsozialistischen Ära resultierenden Belastetheit des Euthanasiebegriffs überwiegend der Begriff Sterbehilfe gebraucht. Dieser führt freilich immer wieder zu medizinischen und rechtlichen Missverständnissen: Denn Sterbehilfe kann ausschließlich Sterbebegleitung, aber auch die Beendigung einer lebenserhaltenden Behandlung oder aber die direkte Tötung eines Patienten durch einen Arzt bedeuten.

Allerdings ist auch der Begriff Euthanasie nicht eindeutig. Etymologisch einen „guten oder schönen Tod“ bezeichnend, war Euthanasie in der Antike als möglichst sanfter und schmerzfreier Sterbeverlauf am Ende eines erfüllten Lebens verstanden. Nie wurde dieses Wort im Sinne eines ärztlichen Eingriffs, welcher das Sterben beschleunigen oder bewirken sollte, aufgefasst. Ein solches Verständnis von Euthanasie ist - von einzelnen Ausnahmen abgesehen - erst am Ende des 19. Jahrhunderts aufgekommen und hat sodann in der nationalsozialistischen Zeit im Rahmen des "Euthanasieprogramms“ zur systematischen Vernichtung „unwerten Lebens “ geführt. Diese strategisch durchgeführte und folglich als unfreiwillige Tötung beziehungsweise präziser als Mord zu kennzeichnende Form der Euthanasie, die auch „soziale Euthanasie“ oder „social killing“ genannt wird, stößt heute auf universelle Ablehnung.

Wenn heute öffentlich über Euthanasie diskutiert wird, dann geht es um die freiwillige Euthanasie, also um die durch einen Arzt herbeigeführte Tötung eines unheilbar kranken, schwer leidenden oder sterbenskranken Menschen auf dessen Verlangen. Mittlerweile gibt es drei europäische Länder, welche ein Gesetz zur Sterbehilfe verabschiedet haben, das es Ärzten unter bestimmten Bedingungen erlaubt, ihre Patienten auf deren Wunsch zu töten: Niederlande (2002), Belgien (2002) und Luxemburg (2009).

Die begrifflichen Kategorien, die das katholische Lehramt im Kontext der Euthanasie vornimmt, sind entlang der Unterscheidung von Töten und Sterbenlassen zu verstehen. Das Töten kennzeichnet die Euthanasie, das Sterbenlassen den Verzicht auf therapeutischen Übereifer und palliative Behandlungsweisen.

\section{Euthanasie}

Im Rückgriff auf die im Jahr 1980 von der Kongregation für die Glaubenslehre veröffentlichte Erklärung zur Euthanasie, definiert Papst Johannes 
Paul II. in seiner Enzyklika Evangelium vitae, die nachfolgend primär herangezogen wird, die „Euthanasie im eigentlichen Sinn“ als eine

„Handlung oder Unterlassung, die ihrer Natur nach und aus bewusster Absicht den Tod herbeiführt, um auf diese Weise jeden Schmerz zu beenden ${ }^{\text {20 }}$.

Entscheidend an dieser Definition ist vor allem die Ebene der Intention, ob also die Herbeiführung des Todes unmittelbar intendiert wird, sei es durch eine Handlung oder Unterlassung. ${ }^{21}$

In der moraltheologischen Literatur existiert bezüglich dieser Ebene die Unterscheidung von direkter oder indirekter Euthanasie oder Sterbehilfe. Sie bezieht sich auf die einer Handlung oder Unterlassung zugrunde liegende Intention der handelnden Person. Dagegen werden die Begriffe Handlung oder Unterlassung der Unterscheidung von aktiver oder passiver Euthanasie oder Sterbehilfe zugeordnet. Diese Ebene der Unterscheidung zielt auf die Beschreibung der Wirkung, welche die (aktive) Handlung oder (passive) Unterlassung selbst hervorbringt - unabhängig von der Intention der handelnden Person. Eine „Euthanasie im eigentlichen Sinn“ ist demnach jegliche direkte Art von Sterbehilfe, wobei es zweitrangig ist, ob die gezielte Herbeiführung des Todes aktiv durch eine Handlung oder passiv durch eine Unterlassung erfolgt. Diese stellt aus katholischer Sicht „eine schwere Verletzung des göttlichen Gesetzes“ dar, „insofern es sich um eine vorsätzliche Tötung einer menschlichen Person handelt, was sittlich nicht zu akzeptieren ist ${ }^{\text {“22. }}$.

\section{Verzicht auf therapeutischen Übereifer}

Die Euthanasie als vorsätzliche Herbeiführung eines vorzeitigen Todes wird abgegrenzt zur

„Entscheidung, auf »therapeutischen Übereifer» zu verzichten, das heißt auf bestimmte ärztliche Eingriffe, die der tatsächlichen Situation des Kranken nicht mehr angemessen sind, weil sie in keinem Verhältnis zu den erhofften Ergebnissen stehen, oder auch, weil sie für ihn und seine Familie zu beschwerlich sind. In diesen Situationen, wenn sich der Tod

\footnotetext{
20 Johannes Paul II., Enzyklika Evangelium vitae, Nr. 65.

21 Dieses entscheidende Merkmal der Intention geht noch deutlicher aus der Definition hervor, die der Katechismus der Katholischen Kirche vorlegt. Es heißt hier: „Die direkte Euthanasie besteht darin, dass man aus welchen Gründen und mit welchen Mitteln auch immer dem Leben behinderter, kranker oder sterbender Menschen eine Ende setzt." (Nr. 2277)

22 Johannes Paul II., Enzyklika Evangelium vitae, Nr. 65.
} 
drohend und unvermeidlich ankündigt, kann man aus Gewissensgründen auf (weitere) Heilversuche verzichten, die nur eine ungewisse und schmerzvolle Verlängerung des Lebens bewirken könnten, ohne dass man jedoch die normalen Bemühungen unterlässt, die in ähnlichen Fällen dem Kranken geschuldet werden. Sicherlich besteht die moralische Verpflichtung sich pflegen und behandeln zu lassen, aber diese Verpflichtung muss an den konkreten Situationen gemessen werden; das heißt, es gilt abzuschätzen, ob die zur Verfügung stehenden therapeutischen Maßnahmen objektiv in einem angemessenen Verhältnis zur Aussicht auf Besserung stehen. Der Verzicht auf außergewöhnliche oder unverhältnismäßige Heilmittel ist nicht gleichzusetzen mit Selbstmord oder Euthanasie; er ist vielmehr Ausdruck dafür, dass die menschliche Situation angesichts des Todes akzeptiert wird. "23

Dieses Zitat spiegelt in moraltheologischer Diktion die indirekt-passive Euthanasie oder Sterbehilfe wider. Hier geht es um die Unterlassung von (weiteren) Therapien oder lebensverlängernden Maßnahmen, wenn diese der Krankheitssituation des Patienten nicht mehr angemessen sind und nur eine „ungewisse und schmerzvolle“ Lebensverlängerung bedeuten würden. Da hierbei nicht der vorzeitige Tod des Patienten intendiert wird, sondern „die menschliche Situation angesichts des Todes akzeptiert wird“ impliziert diese Form der Sterbehilfe keine ethischen Bedenken. Gemäß dem Moraltheologen Eberhard Schockenhoff wäre das

„Sterbenlassen von seiner intentionalen Struktur her nur dann einer Tötungshandlung gleichzusetzen, wenn der Arzt über eine geeignete, angemessene und verhältnismäßige Maßnahme zur weiteren Lebenserhaltung verfügen würde, die er absichtlich unterlässt, um den Tod des Patienten schneller herbeizuführen. Dies darf jedoch beim Sterbenlassen keineswegs als Regelfall unterstellt werden. Der Abbruch einer lebenserhaltenden Behandlung kann vielmehr moralisch vertretbar oder sogar geboten sein, wenn das ursprünglich intendierte Ziel der Heilung nicht mehr erreichbar ist, weil keine aussichtsreiche Therapie zur Verfügung steht oder wenn das Hinausschieben des Todes nur um den Preis unzumutbarer Belastungen für den Patienten erkauft würde. ${ }^{\text {¿24 }}$

Obwohl im Ergebnis die indirekt-passive Sterbehilfe in der Regel einen früheren Todeseintritt zur Folge hat, besteht dennoch ein klarer ethischer Un-

\section{Ibidem.}

24 Eberhard Schockenhoff, Ethik des Lebens, Grundlagen und neue Herausforderungen, Freiburg i.Br. 2009, S. 542. 
terschied zur direkt-passiven Sterbehilfe, die absichtlich auf die rasche Herbeiführung des Todes zielt und so mitunter zentrale Aspekte der Sterbebegleitung vernachlässigt, obwohl diese der Würde des Menschen auch in seinen letzten Tagen und Stunden geschuldet ist. Wo dagegen in indirekt-passiver Form ein menschenwürdiges Sterben unter ärztlicher Fürsorge zugelassen wird, geht es in der Entscheidung zum Verzicht oder Abbruch lebensverlängernder Maßnahmen niemals um die Frage, ob das Leben des schwerkranken Menschen noch lebenswert sei. Vielmehr überprüft der Arzt - oder die Ärztin oder häufig ein Ärzteteam - nur

„die Eignung einer medizinischen Maßnahme, das Behandlungsziel zu erreichen und gelangt dabei zu dem Schluss, dass sie im Blick auf das zusätzliche Leiden, das sie beim Sterbenden verursacht, nicht mehr verhältnismäßig ist. Deshalb kann er sie unterlassen, ohne den Tod des Patienten zu wollen, den zu verhindern nicht mehr in seiner Macht steht. ${ }^{\text {"25 }}$

Eine Lebensverlängerung um jeden Preis entspricht somit im Blick auf Sterbende nicht der katholischen Auffassung, wiewohl ihr das oft vorgehalten wird. Auch wird im Rahmen der indirekt-passiven Sterbehilfe das Selbstbestimmungsrecht des kranken Menschen nicht relativiert. Der Bitte eines unheilbar Kranken, auf (weitere) lebensverlängernde Maßnahmen verzichten zu wollen, haben Ärzte zu entsprechen, da ein Patient niemals Objekt der Behandlung, sondern eigenverantwortliches Subjekt in der Arzt-Patienten-Beziehung ist. „Einen Patienten gegen seinen Willen zu behandeln, ist unabhängig von der Art und dem Stadium seiner Erkrankung weder ethisch vertretbar noch rechtlich zulässig. " ${ }^{26}$ Zudem äußert diese Bitte, die auch in Form einer Patientenverfügung oder durch eine bevollmächtigte Person vorliegen kann, den Wunsch, sterben zu dürfen, nicht aber vom Arzt getötet zu werden.

Interpretationsbedürftig bleibt die katholische Position zum Verzicht auf therapeutischen Übereifer im Hinblick darauf, was - gemäß dem obigen Zitat aus Evangelium vitae - zu den nicht zu unterlassenden „normalen Bemühungen“ gehört, „die in ähnlichen Fällen dem Kranken geschuldet werden“. Als sicher wird diesbezüglich die „moralische Verpflichtung“ angesehen, „sich pflegen und behandeln zu lassen“, wobei „diese Verpflichtung an den konkreten Situationen gemessen werden“ muss. Die hier im Hintergrund stehende Unterscheidung zwischen gewöhnlichen und außergewöhnlichen Mitteln der ärztlichen Behandlung ist aber durch allgemein gültige Kriterien

25 Ibidem.

26 M. de Ridder, Wie wollen wir sterben?, S. 254. 
nicht abschließend festzulegen, da sie eine hohe Gebundenheit an die jeweilige Situation aufweist. Diese Situationsabhängigkeit kann geografischer Art sein, da hierzulande in bestens ausgerüsteten Kliniken etwas ein gewöhnliches Mittel sein kann, was in anderen Kliniken bereits ein außergewöhnliches Mittel wäre. Die Situationsgebundenheit bezieht sich aber ebenso auf den stets individuellen Krankheitsverlauf eines Menschen.

Umstritten ist hier vor allem die Frage, ob am Lebensende die (insbesondere künstliche) Verabreichung von Ernährung und Flüssigkeit immer als ein gewöhnliches Mittel der Lebenserhaltung und folglich als moralisch verpflichtend anzusehen ist. Immerhin existiert auch im alltäglichen Bewusstsein eine tief verwurzelte moralische Intuition, die es als grausam empfinden lässt, einen Patienten verhungern und verdursten zu lassen. Darum scheint gegebenenfalls auch die künstliche Ernährung eine dem Kranken immer geschuldete Fürsorgepflicht zu sein. War die künstliche Ernährung ursprünglich als intensivmedizinische Maßnahme im Sinne eines absehbar vorübergehenden therapeutischen Eingriffs gedacht, so wird sie heute verbreitet auch als Bestandteil der Basispflege bei Schwerstkranken und Sterbenden praktiziert. Doch im Endstadium einer schweren Erkrankung sowie im Sterbeprozess ist die künstliche Ernährung und Flüssigkeitszufuhr anders zu bewerten.

„Denn die Minderung der Aufnahme von Nahrung und Flüssigkeit ist Teil des natürlichen Sterbeprozesses“, der „Wochen oder gar Monate vor dem Tod mit nachlassendem Appetit, allmählicher Gewichtsabnahme, geringerer Aktivität und größerem Schlafbedürfnis einsetzt und fortschreitet, bis der Kranke schließlich in einen Dämmerzustand verfällt und zumeist rasch einer Infektion erliegt. [...] Vieles spricht dafür, dass die Natur auf diese Weise lindernd in den Sterbeprozess eingreift. Es gibt Hinweise darauf, dass bestimmte beim Abbau des Körperfetts anfallende Stoffe, die Ketone, sowie andere Stoffwechseländerungen, die mit verminderter Kalorienaufnahme verbunden sind, einen willkommenen schmerzlindernden Effekt haben. Flüssigkeitsverarmung dämpft zudem die Bewusstseinslage. Sie trägt vermutlich dazu bei, Angstzustände in der Sterbephase zu vermindern. ${ }^{\text {"27 }}$

Vor allem in der Finalphase des Sterbens tragen künstliche Ernährung und Flüssigkeitszufuhr oftmals nicht zur Linderung, sondern umgekehrt zur Steigerung der Schmerzen und des Unwohlbefindens des Patienten bei. Sie würden dann nicht mehr als gewöhnliche, sondern als außergewöhnliche Mittel eingesetzt werden, welche den Sterbeprozess zusätzlich belasten. Der

27 Ibidem, S. 62f. 
Verzicht oder die Einstellung von künstlicher Ernährung und Flüssigkeitszufuhr ist darum in solchen Fällen, die jeweils gewissenhaft zu prüfen sind, als ethisch gebotene Form des Sterbenlassens anzusehen.

Anders als bei sterbenden Menschen urteilt das katholische Lehramt in Bezug auf diese Frage im Blick auf Menschen, die sich im sogenannten ,Wachkoma', medizinisch: im permanenten vegetativen Status, befinden. Da solche Patienten keine sterbenden Menschen sind, stellt „die Verabreichung von Wasser und Nahrung, auch wenn sie auf künstlichen Wegen geschieht, immer ein natürliches Mittel der Lebenserhaltung “28 dar. Andernfalls würde der Tod durch Verhungern und Verdursten absichtlich herbeigeführt werden, was eine „Euthanasie durch Unterlassung “ ${ }^{29}$ wäre.

Im moraltheologischen Diskurs wird diese Thematik zum Teil offener diskutiert. So meint etwa Eberhard Schockenhoff, dass „sich aus dem christlichen Menschenbild keine Verpflichtung zur unbegrenzten Lebenserhaltung um jeden Preis ableiten“ lässt, hält aber zugleich fest, dass ein „solcher Gedanke der kirchlichen Lehrtradition auch im Blick auf die Frage, unter welchen Umständen die Nahrungsaufnahme moralisch geboten ist, durchaus fremd" 30 ist. Deutlicher hat sich der im Jahr 2013 verstorbene Moraltheologe Bernard Fraling geäußert:

\begin{abstract}
„Wenn man sich fragt, soll das sein, eine künstliche Erhaltung vegetativen Lebens mit dem hohen Aufwand einer künstlichen Ernährung und einer Rundumpllege über Jahre hinaus - steht dieser Aufwand zum Ziel in einem angemessenen Verhältnis? Mir fällt es schwer, das zu bejahen und als Behandlung ganz allgemein zu fordern und zu erwarten, dass man dieser Forderung überall entspricht, wo man sich auf das christliche Menschenbild als Grundlage der Behandlung beruft. Ich würde für mich einen solchen Aufwand nicht wünschen. “"
\end{abstract}

Johannes Reiter dagegen meint, dass „das Handeln an Wachkoma-Patienten in mehrfacher Hinsicht ein Handeln in Unsicherheit“ ist und man da-

\footnotetext{
28 Ansprache von Johannes Paul II. an die Teilnehmer des internationalen Fachkongresses zum Thema Lebenserhaltende Behandlungen und vegetativer Zustand: Wissenschaftliche Fortschritte und ethische Dilemmata (20.3.2004), Nr. 4, http:/www.vatican.va/holy_father/ john_paul_ii/speeches/2004/march/documents/hf_jp-ii_spe_20040320_congress-fiamc_ ge.html, abgerufen am 5. Juni 2014.

29 Ibidem.

30 E. Schockenhoff, Ethik des Lebens, S. 399.

31 Bernhard Fraling, „Im Zweifel für das Leben - Künstliche Ernährung bei Patienten im Wachkoma? Ein Diskussionsbeitrag“, in: Peter Neuner, Peter Lüning (Hg.), Theologie im Dialog (FS H. Wagner), Münster 2004, S. 57-70, hier S. 68.
} 
her „eher einen tutioristischen Standpunkt beziehen, sich also für das Leben entscheiden und somit die Ernährung fortsetzen“32 sollte. Dagegen sieht der Mediziner Michael de Ridder diese Unsicherheit als nicht gegeben an, sofern durch gewissenhafte Diagnostik der klinisch ähnliche Zustand minimalen Bewusstseins ausgeschlossen wird. Im eindeutig diagnostizierten Fall eines permanenten vegetativen Status, wo zwar Wachheit, aber de Ridder zufolge mit an Sicherheit grenzender Wahrscheinlichkeit kein Bewusstsein vorhanden ist und dieses auch nicht zurückkehren wird, plädiert er persönlich dafür, trotz fehlender Todesnähe „sich dem Sterben dieser Patienten, die schwerer nicht beschädigt sein könnten, nicht zu verweigern, es sei denn, sie hätten in einer Patientenverfügung anderes hinterlegt oder ihr mutmaßlicher Wille stünde dem entgegen. " ${ }^{33}$

Die strittige Diskussion eines gebotenen Umgangs mit sogenannten Wachkoma-Patienten kann hier nur angezeigt werden. Das kirchliche Lehramt urteilt diesbezüglich in unbedingter Weise zugunsten des Lebens solcher Menschen, zumal die Fachliteratur nach wie vor keine abschließende Stellungnahme zu Diagnose, Therapie und Prognose des Wachkomas erlaubt. ${ }^{34}$

Das in diesem Abschnitt behandelte Thema einer ethisch legitimen oder nicht legitimen Unterlassung von (weiteren) Therapien oder lebensverlängernden Maßnahmen impliziert, dass es der Medizin ab einem gewissen Krankheitsstadium versagt ist, fortgesetzt auf sinnvolle und angemessene Weise zur Heilung des Patienten beizutragen. Das besagt freilich nicht, dass dort, wo medizinisch nichts mehr gemacht werden kann, der Patient aus der Fürsorge ärztlicher Zuwendung entlassen wäre. Geboten ist vielmehr eine Änderung des Behandlungsziels, welche an die Stelle kurativer (= heilender) Maßnahmen die palliative (= lindernde) Sorge um den Patienten treten lässt.

32 Johannes Reiter, „Kein Sterben in Würde. Ist ein Fall Schiavo auch bei uns möglich?“ in: Herder Korrespondenz 59 (5/2005), S. 236-239, hier S. 238.

33 M. de Ridder, Wie wollen wir sterben?, S. 184. M. de Ridder betont, „dass die Abgründe der Medizin nirgendwo sichtbarer werden als im Zustand des permanenten vegetativen Status, in den der Patient [...] ohne die moderne Medizin nicht geraten wäre. Insofern kann und darf man ihn, zweifellos einen lebenden Menschen, dem jeder Respekt gebührt, dennoch als einen medizinischen Artefakt bezeichnen. Und dieser Artefakt soll nun auf unabsehbare Zeit in völliger Abhängigkeit und ohne jede Aussicht auf Teilhabe an menschlicher Gemeinschaft und Umwelt im ,bloßen' Leben gehalten werden? Ist es unangemessen, ein solches Leben erzwungen im Schweigen und in der Isolation - als faktische Misshandlung zu bezeichnen? Ist es wirklich abwegig, so meine Frage an diejenigen, die einem Abbruch lebenserhaltender Maßnahmen bei Patienten im permanenten vegetativen Status niemals zustimmen würden, diesen Zustand als einen zu beschreiben, der bitterer und bedrückender ist als der Tod?", Ibidem, S. $184 f$.

34 Vgl. Wolfram Höfling (Hg.), Das sog. Wachkoma. Rechtliche, medizinische und ethische Aspekte, Münster ${ }^{2} 2007$. 


\section{Palliative Behandlung}

Die dritte begriffliche Kategorie betrifft die „sogenannten «palliativen Behandlungsweisen», die das Leiden im Endstadium der Krankheit erträglicher machen und gleichzeitig für Patienten eine angemessene menschliche Begleitung gewährleisten sollen "35. Diese Art der indirekt-aktiven Sterbehilfe ist ethisch ebenso zulässig, da selbst dann, wenn schmerzlindernde Maßnahmen als letztes Mittel eine Lebensverkürzung oder eine Trübung des Bewusstseins mit sich bringen würden, hierdurch „der Tod nicht gewollt oder gesucht“ wird, sondern man nur „durch Anwendung der von der Medizin zur Verfügung gestellten Analgetika den Schmerz wirksam lindern" ${ }^{36}$ will. Damit soll Menschen auch im Endstadium ihrer Krankheit eine möglichst hohe Lebensqualität erhalten bleiben, denn sie „sollen vor dem herannahenden Tod in der Lage sein, ihren moralischen und familiären Verpflichtungen nachkommen zu können, und sich vor allem mit vollem Bewusstsein auf die endgültige Begegnung mit Gott vorbereiten können“37. Im Anschluss an Papst Pius XII. fordert darum Papst Johannes Paul II., dass „man den Sterbenden nicht ohne schwerwiegenden Grund seiner Bewusstseinsklarheit berauben "38 darf.

Indirekt-aktiv ist diese Form der Sterbehilfe, da vor allem in früheren Jahrzehnten mit der Gabe von Schmerzmitteln und anderen Medikamenten oft eine Lebensverkürzung in Kauf zu nehmen war. Das ist zwar gegenwärtig bei korrekt durchgeführter Behandlung eher die Ausnahme. In den seltenen Fällen, wo durch hoch dosierte schmerzlindernde Medikamente eine Lebensverkürzung auch heute unumgänglich ist, gilt argumentativ unverändert, dass das Ziel der Aufrechterhaltung von Lebensqualität, also die Ermöglichung einer weitgehend schmerzfreien Sterbensphase, als höheres Gut, ja, als humane Pflicht anzusehen ist gegenüber der Aussicht, dem Patienten gegebenenfalls ein kurzfristig längeres Leben unter großen Schmerzen zuzumuten.

Der Wert der Palliativmedizin als eine Behandlungsweise für Patienten, für die eine kurative Behandlung nicht mehr existiert, wird auch in der moraltheologischen Literatur unbestritten anerkannt und mit der Forderung verbunden, diesen im medizinischen System auch verstärkt zu etablieren. Denn nach wie vor sind unsere Krankenhäuser, wenn man „von den wenigen

\footnotetext{
35 Johannes Paul II., Enzyklika Evangelium vitae, Nr. 65.

36 Ibidem.

37 Ibidem.

38 Ibidem.
} 
Ausnahmen absieht, der Stein und Stahl gewordene Gegenentwurf zu dem, was ein Mensch am Ende seines Lebens braucht ${ }^{\text {“39 }}$ Die extrem ausdifferenzierte Spezialisierung der modernen Medizin führt oft dazu, dass nur von der Person abgespaltene Krankheiten behandelt werden, der Patient als Mensch in seiner Ganzheit aber aus dem Blick gerät. De Ridder sieht darum in der erst in den späten 1980er Jahren als eigene medizinische Disziplin konzipierten - Palliativmedizin das Potential schlummern,

„,in unserer Medizin einen Kulturwandel zu bewirken, ja, sie in geradezu revolutionärer Weise zu verändern: Weg von einer Medizin, in deren Mittelpunkt die Krankheit und nicht der von ihr erfasste Mensch steht! Weg von der Lebenserhaltung um jeden Preis! Weg von einer Medizin, der Empathie ein Fremdwort ist! Weg von einer Ärzteschaft, die dem irrigen Gedanken aufsitzt, dass ein sterbender Mensch gleichzusetzen sei mit einer Niederlage ärztlichen Könnens! Weg von Ärzten, die es scheuen, sich mit der eigenen Sterblichkeit auseinanderzusetzen! Stattdessen hin zu einer Medizin, [...] in deren Mittelpunkt der kranke Mensch und nicht das kranke Organ steht! Hin zu einer Medizin, die zwischen sinnvoller Lebensverlängerung und qualvoller Sterbeverzögerung zu unterscheiden vermag! Hin zu einer Medizin, die nicht nur weiß, sondern auch annimmt und ernst nimmt, dass ihr palliativer Auftrag ihr zentrales Anliegen ist und sein muss, ihr kurativer Auftrag dagegen nachgeordnet ist. “ 40

Dieses Potential des palliativen Auftrags der Medizin ist bei Weitem noch nicht ausgeschöpft und ein tiefgreifender Kulturwandel der Medizin längst noch nicht vollzogen, wiewohl es zahlreiche Aufbrüche in diese Richtung gibt. Diese sind freilich notwendig, wenn es um ein Sterben in Würde geht.

Doch ist nicht der Begriff Würde selbst von einem merkwürdigen Widerspruch gekennzeichnet, wenn zum einen unter der Würde des Menschen dessen Recht auf Selbstbestimmung verstanden wird, was auch einen selbstbestimmten Tod impliziert, zum anderen aber eben diese Auffassung, wie etwa seitens der katholischen Kirche, als völlig unvereinbar mit der menschlichen Würde angesehen wird. Ist für die letztere Sicht nur der theologische Ausgangspunkt verantwortlich? Hans Küng zufolge schließt dieser aber die erstgenannte Würdevorstellung keineswegs aus.

39 M. de Ridder, Wie wollen wir sterben?, S. 228.

40 Ibidem, S. 221. 


\section{Welche Würde?}

Ohne hier auf Details eingehen zu können, kann vereinfacht festgehalten werden, dass in der Debatte um ein menschenwürdiges Sterben die Würdevorstellung auf zumindest zwei Ebenen artikuliert wird: ${ }^{41}$

Auf einer ersten Ebene ist von einer absoluten Würdevorstellung die Rede, welche unsere abendländische Geschichte geprägt hat. Sie meint einen inneren, unbedingten Wert, der allen Menschen aufgrund ihres Menschseins gleichermaßen zukommt - unabhängig von Leistung, Amt oder Ansehen sowie unabhängig von Geschlecht, Alter, Rasse oder Religion. Demgemäß ist Würde unteilbar, unverrechenbar und unantastbar. In ethischer Hinsicht folgt daraus

„das strikte Verbot, den Wert der Menschenwürde zu teilen, zu verrechnen oder anzutasten. Als innerer, unbedingter Wert bleibt Menschenwürde somit jeder äußeren, bedingungsabhängigen Bewertung durch den Menschen entzogen. Die Menschenwürde bedarf daher der Achtung, denn Achtung besagt Anerkenntnis von Würde, die sich menschlicher Verfügbarkeit entzieht. ${ }^{“ 42}$

Diese a priori anzuerkennende Würde wird theologisch mit der Gottebenbildlichkeit des Menschen (Gen 1,27), welche die Fähigkeit des Menschen zur Gemeinschaft mit Gott impliziert, begründet. Philosophisch wird sie von Immanuel Kant nicht in der Gottfähigkeit des Menschen, sondern in dessen Moralfähigkeit fundiert. Da für ihn Moralität die Bedingung dafür ist, als Selbstzweck existieren zu können und nicht bloß als Mittel zu irgendeinem Zweck, kommt Würde primär der Sittlichkeit zu und damit jedem Menschen:

„Nun ist Moralität die Bedingung, unter der allein ein vernünftiges Wesen Zweck an sich selbst sein kann. [...] Also ist Sittlichkeit und die Menschheit, sofern sie derselben fähig ist, dasjenige, was allein Würde hat. “43

Hierbei ist zu beachten, dass das, was den Menschen als Menschen charakterisiert, nicht die Moralpraxis, sondern die Moralfähigkeit ist, die wie-

${ }^{41}$ Vgl. hierzu: Arnd Pollmann, „Würde nach Maß“ in: Deutsche Zeitschrift für Philosophie 53 (2005), S. 611-619; Ralf Stoecker. (Hg.), Menschenwürde. Annäherung an einen Begriff, Wien 2003.

42 Gerhard Marschütz, theologisch ethisch nachdenken. Band 1: Grundlagen, Würzburg ${ }^{2} 2014$, S. 245.

43 Immanuel Kant, Grundlegung zur Metaphysik der Sitten, BA 66, zitiert nach der Werkausgabe, Band 7. Hg. v. Wilhelm Weischedel, Frankfurt ${ }^{18} 2008$. 
derum der Vernunftfähigkeit bedarf. Wollte man die Menschenwürde von aktuellen Vollzügen der Vernunft oder einer guten moralischen Praxis abhängig machen, wären Menschen in ihrer Würde ungleich. Die Würde ist aber nach Kant strikt als Achtung der Menschheit in jedem Menschen zu verstehen. Folglich ist die Würde als innerer, unbedingter Wert nicht durch äußere Faktoren bewertbar, nicht gegenüber anderen Gütern abwägbar und somit auch nicht graduierbar, wonach sie etwa am Anfang oder Ende des menschlichen Lebens weniger oder gar nicht mehr gegeben wäre. ${ }^{44}$

Auf einer zweiten Ebene wird Würde nicht a priori anerkannt, sondern a posteriori zuerkannt, wobei die Bedingungen der Zuerkenntnis von Würde sehr unterschiedlich formuliert sein können - von der Gesellschaft ebenso wie von jedem Einzelmenschen. Jedenfalls bezeichnet Würde hier kein Merkmal, das allen Menschen aufgrund ihres Menschseins gleichermaßen zukommt, sondern das, was Menschen aufgrund bestimmter äußerer Bedingungen je unterschiedlich zukommt, wobei sie manchen Menschen auch nicht zukommen muss und folglich Würde nur eine Teilmenge der Menschheit umfasst. So kann beispielsweise Embryonen und Feten oder Demenz- und Sterbenskranken in einer bestimmten Gesellschaft die Würde noch nicht oder nicht mehr zuerkannt werden. Demnach kann Würde erworben, aber auch verloren werden. Deren Vorhandensein in Abhängigkeit von diversen äußeren Faktoren kann auch individuell interpretiert werden. Im Blick auf das eigene Sterben kann Würde im Kontrast zu einer bestimmten Vorstellung von Lebensqualität begriffen werden. Würde wird dann davon abhängig, inwieweit man noch einigermaßen im Vollbesitz geistiger und körperlicher Fähigkeiten ist und ein selbstbestimmtes Leben führen kann. Die mit der Würdevorstellung verbundene Möglichkeit der Selbstbestimmung wird dann in gesunden Tagen als Recht postuliert, den in aussichtsloser Krankheit oder in der letzten Lebensphase befürchteten geistigen, psychischen oder körperlichen Verlust dieser Möglichkeit nicht passiv hinnehmen zu müssen, sondern aktiv beenden zu können.

In dieser Vorstellung ist Würde kein innerer Wert, der sich jeder äußeren Bewertung entzieht und darum unbedingt zu achten ist, sondern ein $\ddot{u} u ß e r e r$ Wert, der erst relativ zur Lebensqualität gegeben ist und darum im schlimmsten Falle gar nicht mehr vorhanden sein kann. Beiden Würdevorstellungen liegen letztendlich unterschiedliche anthropologische Prämissen zugrunde. ${ }^{45}$

44 Für Kant ist darum jeder Mensch als Person anzuerkennen, auch der Embryo, oder wie Kant sagt, das durch den menschlichen Akt der Zeugung Erzeugte. Vgl. dazu G. Marschütz, theologisch ethisch nachdenken. Band 2: Handlungsfelder, Würzburg 2011, S. 199.

45 Vgl. Ruth Scholz, Die Diskussion um die Euthanasie. $\mathrm{Zu}$ den anthropologischen Hintergründen einer ethischen Fragestellung, Münster 2002. 


\begin{abstract}
„Die an äußeren Faktoren der Lebensqualität bemessene Würde geht von einem empirischen Menschenbild aus. Würde wird hier zum relativen Wert, da dieser an das empirisch nachweisbare Vorhandensein von Eigenschaften, Fähigkeiten oder Qualitäten des Menschen geknüpft wird. In einer solchen Sicht wird der Mensch tendenziell auf seinen gesellschaftlichen Nutzwert reduziert. Dagegen steht die Vorstellung einer absoluten Würde in der Tradition des Christentums wie auch in der Tradition der Stoa und des Philosophen Kant. Diese setzt ein metaphysisches Menschenbild voraus, da der Mensch nicht nur ein empirisches Wesen ist, sondern ihm auch die alles Empirische überschreitende Sinnfrage eigen ist, die ihn unbedingt beansprucht. Hierin erfährt er sich trotz allem (bruchstückhaften) Verstehen bleibend als Frage, somit sich selbst (wie auch anderen) entzogen und unverfügbar. Dem entspricht, dass Würde als ein innerer, unverfügbarer Wert vorgestellt wird, der um des Menschen willen unbedingt zu achten, zu schützen und zu gewähren ist. “46
\end{abstract}

Beide Ebenen der Würdevorstellung, die metaphysische und die empirische, sind in der abendländischen Tradition integrativ begriffen worden, wobei aber das empirische Würdeverständnis für das Verstehen von Würde „nicht primär, sondern sekundär“ ${ }^{47}$ war. Es stellte somit eine

„hinzukommende, nicht aber fundamentale Interpretation von Würde dar. Anders gesagt: Es hat Anteil an der allen Menschen zukommenden Würde, aber Würde gründet nicht darin. " ${ }^{48}$

In den gegenwärtigen Plädoyers für ein selbstbestimmtes Sterben hat sich aber das empirische Würdeverständnis als primäre Interpretation von Würde etabliert, völlig abgekoppelt von metaphysischen Dimensionen. Daraus entspringt oft ein verabsolutiertes Autonomieverständnis, das vorgestellt wird „als Vermögen eines unabhängigen und isolierten Subjekts, das frei von der Beeinflussung durch andere handelt und entscheidet ${ }^{\text {“49 }}$. Zweifellos wird ein solches Verständnis besonders durch moderne Lebensbedingungen gefördert, in denen Menschen ihre soziale Positionierung mehr denn je selbst bestimmen und behaupten müssen. Hierbei wird implizit

46 G. Marschütz, theologisch ethisch nachdenken, Band 2, S. 290.

47 Werner Wolbert, „Menschenwürde als Anspruch und Beschränkung“ in: Kurt Seelmann (Hg.), Menschenwürde als Rechtsbegriff, ARSP-Beiheft 101, Stuttgart 2004, S. 162-174, hier S. 167.

48 G. Marschütz, theologisch ethisch nachdenken, Band 1, S. 247.

49 E. Schockenhoff, Ethik des Lebens, S. 538. 
eine möglichst lebenslang gegebene geistige, psychische und körperliche Funktionsfähigkeit vorausgesetzt, die keineswegs selbstverständlich ist. Zugleich erweist sich der irgendwann unausweichliche Zerfall dieser Fähigkeit in schwerer Krankheit und zuletzt im Sterben als Bedrängung, auf die der Mensch inmitten des Lebens mit Verdrängung reagiert, ebenso aber mit dem Drängen danach, diesen unabwendbaren Zerfall nicht als Widerfahrnis aushalten zu müssen. Der Tod soll darum entweder nach einem langen, erfüllten Leben möglichst schmerzlos und plötzlich eintreten oder, wenn dies nicht der Fall ist - immerhin sterben über 90\% der Menschen infolge länger andauernder Krankheiten, die Wochen, Monate und auch Jahre andauern können -, auf eigenes Verlangen mit ärztlicher Hilfe und damit nach eigenen Vorstellungen erfolgen können.

Obzwar diese an die Würdevorstellung gebundene Form der Selbstbestimmung im Kontext moderner Lebensbedingungen gut nachvollziehbar ist, so trifft sie dennoch nur die halbe Wahrheit. Ganz wesentlich zum Menschsein gehört nämlich ebenso, ein bedürftiges, auf andere angewiesenes Lebewesen zu sein. Bereits der Anfang jedes Menschen im Akt seines Gezeugt-Werdens, stellt für diesen selbst ein passives Geschehen dar, für das er keine Verantwortung trägt. Und das gesamte weitere Leben hindurch existieren er in einer Vielfalt von Relationen - angefangen von den Eltern über den Schulbesuch bis hin zum Freundeskreis, der Gründung einer Familie und den Kollegen am Arbeitsplatz - die sein Leben nachhaltig bestimmen und erst ermöglichen, sich selbst finden und bestimmen zu können. Selbstbestimmung erwächst aus Fremdbestimmung, wobei diese in der Regel eben nicht negativ zu begreifen ist und somit auch keine menschenunwürdige, sondern eine das Menschsein ermöglichende Bedingung darstellt. Letztlich trifft das auch auf die liebende Beziehung Gottes zu den Menschen zu, die im tiefsten Sinn des Wortes erst Leben verleiht. Wer dagegen in absoluter Weise sein Leben als Besitz begreift und daraus ein Selbstbestimmungsrecht ableitet, der blendet zentrale Aspekte des Menschseins aus.

Da sich am Lebensende die Abhängigkeit des menschlichen Daseins in besonderer Weise zeigt, erfordert

„ein menschenwürdiges Sterben mehr als bloßen Respekt vor einer angeblich unbeeinflussten Selbstbestimmung des Sterbenden. Menschenwürdiges Sterben ist überhaupt nur unter der Bedingung möglich, dass personale Beziehungen und menschliche Nähe zum Sterbenden aufrecht erhalten werden. " 50

$50 \quad$ Ibidem. 
Wo ein Mensch sich in seiner letzten Lebensphase der personalen Nähe anderer gewiss sein darf, wird er trotz aller hinzukommenden misslichen Formen der Abhängigkeit den Wunsch nach Tötung, wenn überhaupt, nur selten äußern - zumindest kaum je in beständiger Weise, wie Erfahrungen aus der Palliativmedizin und der Hospizbewegung zeigen. Die Abhängigkeit des nicht oder nur selten geäußerten Todeswunsches vom Vorhandensein guter menschlicher Beziehungen wird oft viel zu wenig in der Diskussion um die direkt-aktive Sterbehilfe beachtet. Es ist daher entscheidend, dass sterbenskranken Menschen ihrem bevorstehenden physischen Tod nicht der soziale und psychische Tod vorausgeht. Entscheidend ist freilich auch, dass die Formen der indirekten Sterbehilfe genutzt werden, damit jeder Mensch möglichst seinen eigenen Tod finden kann und nicht eine unangebrachte Verzögerung seines Sterbens befürchten muss. Die Anwendung dieser Formen ist freilich unausweichlich auch mit Grauzonen des Handelns verbunden, die im jeweiligen Einzelfall bestmöglich durch ein Konsilium von Experten (Ärzte, Ethiker ...) einer Klärung zuzuführen sind. Hierfür eine Legalisierung der Tötung auf Verlangen unter bestimmten Bedingungen fordern zu wollen, würde diese Grauzonen nur verlagern. Das zeigt die Praxis in den Niederlanden und in Belgien, wo etwa 20-25\% aller im Rahmen des Sterbehilfegesetzes durchgeführten Tötungen ohne Einwilligung der Patienten erfolgen und in etwa 50\% der Fälle zwischen der Todeswunsch-Äußerung und der Tötung nur wenige Tage oder Stunden liegen, was die gesetzlich geforderte Beständigkeit und Ernsthaftigkeit des Wunsches nicht glaubhaft nachvollziehen lässt. ${ }^{51}$ Eine Legalisierung würde die Grenzlinie zwischen Töten und Sterbenlassen noch mehr verwischen und eine deutliche Absenkung der Hemmschwelle zur Tötung bewirken. ${ }^{52}$

Dennoch: Selbst ein Umgang der personalen Nähe mit Sterbenden bewahrt nicht immer davor, dass diese ihre Situation in vielerlei Hinsicht als entwürdigend oder gar würdelos erfahren. Der Vorgang des Sterbens ist in der Regel mühsam und vermittelt oft nur wenig davon, was Würde in ihrer empirischen Qualität ausmacht. Vielleicht sollte man darum nur zurückhaltend von einem »Sterben in Würde« sprechen. Das Ideal eines würdigen Sterbens lässt

51 Vgl. Gerhard Baader, „Aktive Sterbehilfe in den Niederlanden heute - Analogie zur NS-Euthanasie?" in: Viola Schubert-Lehnhardt (Hg.), Medizin-Ethik- quo vadis, Gerbstedt 2002, S. 87-98; Simon Alfred, „Die Praxis der aktiven Sterbehilfe in den Niederlanden und Belgien“ in: Dietrich Kettler et al. (Hg.), Selbstbestimmung am Lebensende, Göttingen 2006, S. $115-123$.

52 Hinsichtlich der Konsequenzen, die eine Legalisierung der Tötung auf Verlangen mit sich bringen würde vgl. Theo R. Payk, Der beschützte Abschied. Streitfall Sterbehilfe, München 2009 . 
eine Sanftheit und Friedlichkeit des Sterbens assoziieren, das der Realität des Sterbens häufig nicht entspricht. Zweifellos ist aber ein würdiger Umgang mit Sterbenden geboten, der diesen oft erst ermöglicht, mit entwürdigenden Situationen zurechtzukommen, und so die Würde des Sterbenden bis zuletzt wahrt.

\section{Sterben als Leben in Würde ermöglichen}

Der Umgang mit sterbenden Menschen sagt viel über die Humanität einer Gesellschaft aus. Doch die moderne Gesellschaft „hat den Tod ausgebürgert " ${ }^{\text {"53 }}$ in Pflegeheime und Krankenhäuser, um möglichst ungestört funktionieren zu können. Vor allem das „Leben der Großstadt wirkt so, als ob niemand mehr stürbe" 54 . So verwundert es nicht, dass unsere Gesellschaft kaum mehr tragfähigen Antworten auf die radikale Infragestellung des Lebens durch den Tod bereitstellt. Solange sich moderne Kulturen und in ihnen der medizinische Fortschritt primär verstehen als „ausgeklügelter Protest gegen das Geschick, dass wir sterblich sind“"55, werden sie solche Antworten nicht finden - es sei denn in der freiwilligen Euthanasie, worin dieser Protest gegen die Entmächtigung des Lebens in die Ermächtigung zum Töten transformiert wird.

Eine Ethik, die der unantastbaren Menschenwürde verpflichtet ist, wird permanent Protest gegen die zunehmende Verdrängung des Umgangs mit Sterben und Tod einlegen, da die Würde des Menschen auch in der Bereitschaft besteht, Sterben und Tod als natürliches Geschehen zu akzeptieren. Eine solche Ethik wird sich für eine humane Sterbebegleitung einsetzen und hierfür entsprechende soziokulturelle Bedingungen einmahnen. Denn wenn aus medizinischer Sicht für schwerkranke und sterbende Menschen nichts mehr getan werden kann, bleibt immer noch sehr viel zu tun. Gemeint ist nicht die Hilfe zum Sterben wie die direkt-aktive Sterbehilfe und Suizidbeihilfe, welche nur auf den ersten Blick als die Lösung erscheinen, solchen Menschen wirksam helfen zu können. Einem humanen Blick geht es um Hilfe im Sterben, die palliativmedizinischer Betreuung und intensiver menschlicher Zuwendung bedarf, um Menschen die Annahme ihres Sterbens zu ermöglichen. Allein dieser Blick „wird der körperlichen und seelischen Not schwerstkranker Menschen gerecht, ohne [...] die moralischen Grundsätze des Tötungsverbotes und die Unterscheidung von Töten und Sterbenlassen außer Kraft" ${ }^{\text {"56 }}$ zu setzen.

53 Philippe Ariès, Geschichte des Todes, München ${ }^{8} 1997$, S. 716.

54 Ibidem.

55 Zygmunt Bauman, Tod, Unsterblichkeit und andere Lebensstrategien, Frankfurt a.M. 1994, S. 65.

56 E. Schockenhoff, Ethik des Lebens, S. 541. 
Ein derart humaner Blick wird die palliative Sorge stärken, welche sich umfassend der Linderung von sowohl physischen wie auch psychischen, sozialen und spirituellen Leiden widmet, um so Menschen auf ihrem letzten Lebensweg ein gute Lebensqualität und eine möglichst selbstbestimmte Annahme des Sterbens ermöglichen zu können. Im Zentrum steht der Sterbende in all seinen Dimensionen und mit all seinen in der letzten Lebensphase aufbrechenden Fragen und Ängste, mit denen er sich nicht selbst überlassen sein will. Eine solche umfängliche Begleitung von kranken Menschen erfordert nicht nur in physischer Schmerzbekämpfung gut ausgebildete Ärzte, sondern ebenso die Einbeziehung (und gegebenenfalls Betreuung) von Familienangehörigen sowie von Pflegepersonal, Sozialarbeitern, Psychologen, Physiotherapeuten und Seelsorgern. Hierzu bedarf es auch des Nachdenkens über neue Modelle von Sterbebegleitung, etwa über sogenannte „Compassionate Communities“, wo vor Ort in nachbarschaftlicher Hilfe und unter punktueller Einbeziehung von Experten Sterbende betreut werden. ${ }^{57}$ Auch kirchliche Gemeinden wären hier besonders herausgefordert, aktiv zu werden.

Palliative Betreuung wurzelt in den bereits im Mittelalter existierenden und von der Ärztin Cicely Saunders vor einigen Jahrzehnten wieder neu ins Leben gerufenen Hospizen, deren Grundidee Sanders prägnant so umschreibt:

„Du bist wichtig, einfach weil du bist. Du bist bis zum letzten Augenblick deines Lebens wichtig. Und wir werden alles tun, damit du nicht nur in Frieden sterben, sondern auch LEBEN kannst - bis zuletzt. “"58

Palliative Sorge ist aber niemals eine Einbahnkommunikation. Sie ermöglicht Sterbenden „ihre letzte Reifung“ und hierin die Fähigkeit Loslassen-zu-Können. ${ }^{59}$ Zugleich schenkt sie den vorerst noch am und im Leben bleibenden gewandelte Maßstäbe dessen, was im Leben wirklich zählt: Nicht die Existenzweise des Habens, sondern des Vertrauen-Dürfens und Eingebettet-Seins in Liebe. Die Schriftstellerin Hilde Domin schreibt in ihrem Gedicht „Unterricht“, das sich in ihrem Buch Nur eine Rose als Stütze findet:

Jeder der geht

belehrt uns ein wenig

über uns selber.

\footnotetext{
57 Vgl. hierzu Reimer Gronemeyer, Andreas Heller, In Ruhe sterben. Was wir uns wünschen und was die moderne Medizin nicht leisten kann, Düsseldorf 2014.

58 http://www.palliativ.or.at/, abgerufen am 5. Juni 2014.

59 Vgl. Monika Renz, Zeugnisse Sterbender. Todesnähe als Wandlung und letzte Reifung, Paderborn ${ }^{2} 2008$.
} 
Kostbarster Unterricht

an den Sterbebetten.

Wie sehr prägt dieser kostbarste Unterricht unser Leben? Vermutlich wäre er in unser Bildungs- oder besser: Lebensprogramm erst noch aufzunehmen.

Die in der palliativen Betreuung zum Ausdruck gelangende unbedingte Wertschätzung jedes Menschen, auch im Sterben, das Teil des Lebens ist, entspricht einer zutiefst christlichen Geisteshaltung. Aus ihr heraus entsteht eine Alternative zur direkt-aktiven Sterbehilfe, die es auf allen Ebenen zu forcieren gilt. Niemand soll sagen, dass das finanziell nicht leistbar ist, und dabei verschwiegen wird, „dass mindestens 20\% aller Gesundheitsausgaben für unnütze Behandlungen verschwendet werden "60. Stattdessen gilt es zu kommunizieren: Das Nein der katholischen Kirche zur Euthanasie ist ein Ja zum Leben - bis zuletzt. Nur ein solches Ja wahrt die Würde von Sterbenden und befruchtet einen würdevollen Umgang im Leben der Menschen.

60 M. de Ridder, Wie wollen wir sterben?, S. 89. 